Proc. Instn Civ. Engrs, Part 2, 1991, 91, Sept., 609-615

DISCUSSION ON PAPER 9441 STRUCTURAL ENGINEERING GROUP

\title{
Minimum reinforcement solution for flat slabs
}

\author{
M. Z. Ahmad and T. K. Datta
}

\section{R. E. Melchers, University of Newcastle, Australia}

There is a danger in applying the approach used by the Authors that idealistic assumptions will be made. The solution they propose is limited by considering only bending action and by idealizing the columns as point supports. Neither of these conditions are met in practice. It would be of interest to know how the optimal solution changes when the local shear effects around the point columns are considered. In practice, of course, the high shears existing around columns are often catered for by using drop-panels. It would, therefore, be interesting to know what the effect is on the optimal solution of finite sized drop-panels and finite columns. An idealized solution based on completely curtailed reinforcement has been given for the bending part of this problem. ${ }^{16}$

\section{Dr Ahmad and Professor Datta}

We agree that the minimum reinforcement solution for flat slabs is presented on the basis of certain idealistic assumptions. However, the assumption of point supports for flat slabs is not uncommon in plastic analysis: e.g. in yield-line analysis. The plastic strengths of the flat slab panels are often obtained with the help of yield-line analysis, without considering the effects of load shear around the finite columns or drop panels.

35. The assumption of point supports for the flat slab was necessary in order to achieve the exact optimum solution using the static-kinematic approach proposed by Rozvany. If finite columns or drop panels are considered, it is not possible to obtain a kinematically admissible displacement field that satisfies the optimality criterion (equations (1) and (2) of the Paper). As a consequence, exact optimum solution is not possible for such cases. However, an upper bound minimum reinforcement solution can be presented, using only statical consideration: i.e. using equation (3) of the Paper. The minimum moment capacity volume thus obtained does not differ significantly from the solution obtained using point support idealization. ${ }^{17}$

\section{Flat slab on finite column supports}

36. An interior panel of flat slab floor system is shown in Fig. 8. The slab panel is supported on rectangular column supports of finite dimension $2 a L \times 2 b L$. It is difficult to obtain a kinematically admissible displacement field that satisfies the optimality conditions (equations (1) and (2) of the Paper) for such slab panels. Therefore, it is not possible to obtain an exact minimum reinforcement solution in this case. An upper bound minimum reinforcement solution can, however, be furnished by specifying a statically admissible safe moment field within the slab.

Paper published: Proc. Instn Civ. Engrs, Part 2, 1989, 87, Dec., 487-500. 


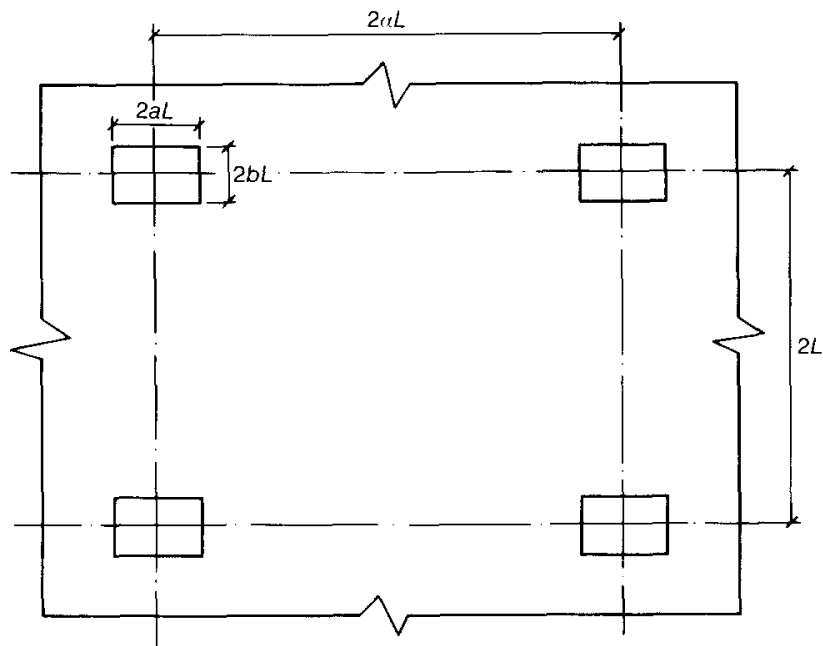

(a)

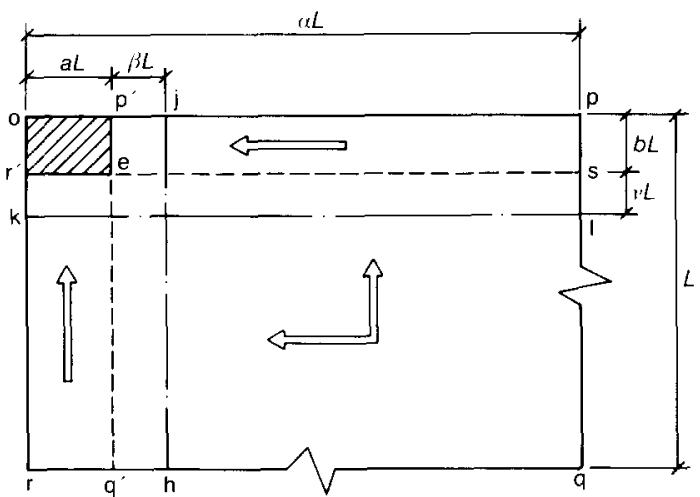

(b)

Fig. 8. Flat slab with finite column supports: (a) typical interior panel of flat slab; (b) load dispersion from quarter panel to column supports

The upper bound on the minimum moment capacity volume will be given by

$$
\phi_{\mathrm{upp}}=\iint_{A}\left(\bar{M}_{x}^{+}+\bar{M}_{y}^{+}+\bar{M}_{x}^{-}+\bar{M}_{y}^{-}\right) \mathrm{d} x \mathrm{~d} y
$$

in which $\bar{M}_{x}^{+}, \bar{M}_{y}^{+}$, etc., are the moment capacities of the $X$ and $Y$ strips of the slabs. These moment capacities are obtained from the maximum moments developed within the strips as a result of the assumed moment field.

37. The load dispersion takes place without twisting moment: i.e. load is transferred by strip action (Fig. 8). For $X$ strips in the region r'sqr, the line of zero 610

Downloaded by [] on [26/04/23]. Copyright @ ICE Publishing, all rights reserved. 
shear lies along sq, and the line of zero moment lies along jh. The $X$ strips are supported by $Y$ strips over the region rr'eq'. Similarly, $Y$ strips in the region p'pqq' $^{\prime}$ are supported by $X$ strips over the region p'pse. For $Y$ strips, the lines of zero shear and zero moment lie along $\mathrm{q}^{\prime} \mathrm{q}$ and $\mathrm{kl}$ respectively. The $Y$ strips in the region ${ }^{\prime \prime} \mathrm{r}^{\prime}{ }^{\prime} \mathrm{e}$, and the $X$ strips in the region $\mathrm{p}^{\prime}$ pse transfer the load finally to the finite area of the column support. The moment field and the corresponding moment capacities of the strips are shown in Fig. 9.

$X$ strips (2-2)

38. With reference to Figs 8 and $9(a)$ and (b), the expression for the maximum hogging and sagging moments of a strip of unit width are

$$
\begin{aligned}
& M_{x}=\frac{(\alpha-a-\beta)^{2} L^{2}}{2} K p \\
& \bar{M}_{x}=\left[(\alpha-a-\beta) \beta L^{2}+\frac{\beta^{2} L^{2}}{2}+\frac{(\alpha-a) a}{2}\right] K p
\end{aligned}
$$

in which $K p$ is the fraction of the load carried by the $X$ strips. With the yield moment capacity of the strip (Fig. 9(c)), the moment capacity volume of the region $r^{\prime} \mathrm{rq}^{\prime} \mathrm{e}$ (Fig. 8(b)) in the $x$ direction is obtained as

$$
\phi_{x 1}=\left[M_{x} \alpha L+\bar{M}_{x}(a+\beta) L\right](1-b) L
$$

$Y$ strips (2-2)

39. The maximum hogging and sagging moments of a strip of unit width (Figs 8 (b) and 9) are given by

$$
\begin{aligned}
& M_{y}=\frac{(1-b-v)^{2} L^{2}}{2}(1-K) p \\
& \vec{M}_{y}=\left[(1-b-v) v L^{2}+\frac{v^{2} L^{2}}{2}+\frac{(1-b) b}{2}\right](1-K) p
\end{aligned}
$$

40. With the yield moment capacity of the strip (Fig. 9(j)), the moment capacity volume of the region p'pqq' (Fig. 8(b)) in the $y$ direction is obtained as

$$
\phi_{y 1}=\left[M_{y} L+\bar{M}_{y}(b+v) L\right](\alpha-a) L
$$

$X$ strips $(1-1)$

41. The $X$ strips (1-1) transfer the load to the column support (Fig. 9(e)). The maximum hogging and sagging moments developed in a strip of unit width are given by

$$
\begin{aligned}
& M_{x}^{\prime}=\left[\frac{(1-b)}{2}(1-K)+1\right] \frac{(\alpha-\beta-a)^{2}}{2} p L^{2} \\
& \bar{M}_{x}^{\prime}=\left[\frac{(1-b)}{b}(1-K)+1\right]\left[(\alpha-\beta-a) \beta+\frac{\beta^{2}}{2}+\frac{(\alpha-a) s}{2}\right] p L^{2}
\end{aligned}
$$




\section{DISCUSSION}

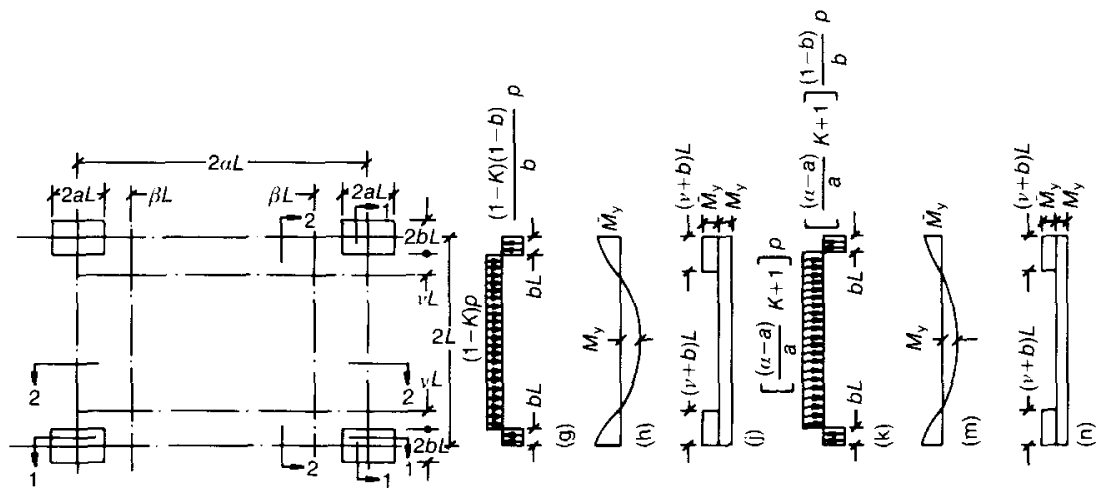

Kp

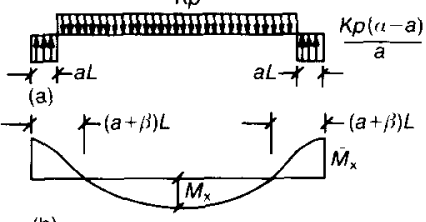

(b)

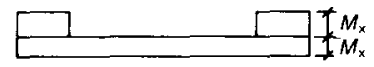

(c)

$\left[\frac{(1-K)(1-b)}{b}+1\right] p$
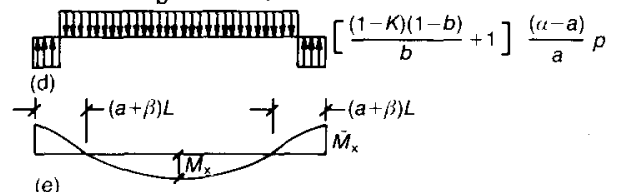

(e)

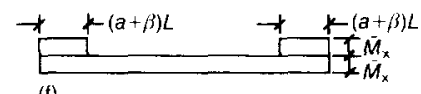

(f)

Fig. 9. Moment field and moment capacities of strips: (a) load intensity in strip 2-2; (b) bending moment in strip 2-2; (c) yield moment capacity of strip 2-2; (d) load intensity in strip $1-1 ;(e)$ bending moment in strip $1-1 ;(f)$ yield moment capacity of strip $1-1 ;(g)$ load intensity in strip 2-2; (h) bending moment in strip $2-2 ;(j)$ yield moment capacity of strip 2-2; $(k)$ load intensity in strip $1-1 ;(m)$ bending moment in strip $1-1 ;(n)$ yield moment capacity of strip $1-1$ 
42. With the moment capacity volume of the strips (Fig. $9(f))$, the moment capacity volume $V_{x 2}$ of the region $\mathrm{r}^{\prime}$ ops (Fig. 8(b)) in the $x$ direction is expressed as

$$
\phi_{x 2}=\left[M_{x}^{\prime} \alpha L+\bar{M}_{x}^{\prime}(a+\beta) L\right] b L
$$

$Y$ strips (1-1)

43. Transfer of the load to the column supports and the consequent bending moments are shown in Fig. $9(\mathrm{k})-(\mathrm{m})$. The maximum hogging and sagging moments for the $Y$ strips are given by

$$
\begin{aligned}
& M_{y}^{\prime}=\left\{\left[\frac{(\alpha-a)}{a}(K+1)\right] \frac{(1-b-v)^{2}}{2}\right\} p L^{2} \\
& \bar{M}_{y}^{\prime}=\left\{\left[\frac{(\alpha-a)}{a} K+1\right]\left[(1-b-v) v+\frac{v^{2}}{2}+\frac{(1-b) b}{2}\right]\right\} p L^{2}
\end{aligned}
$$

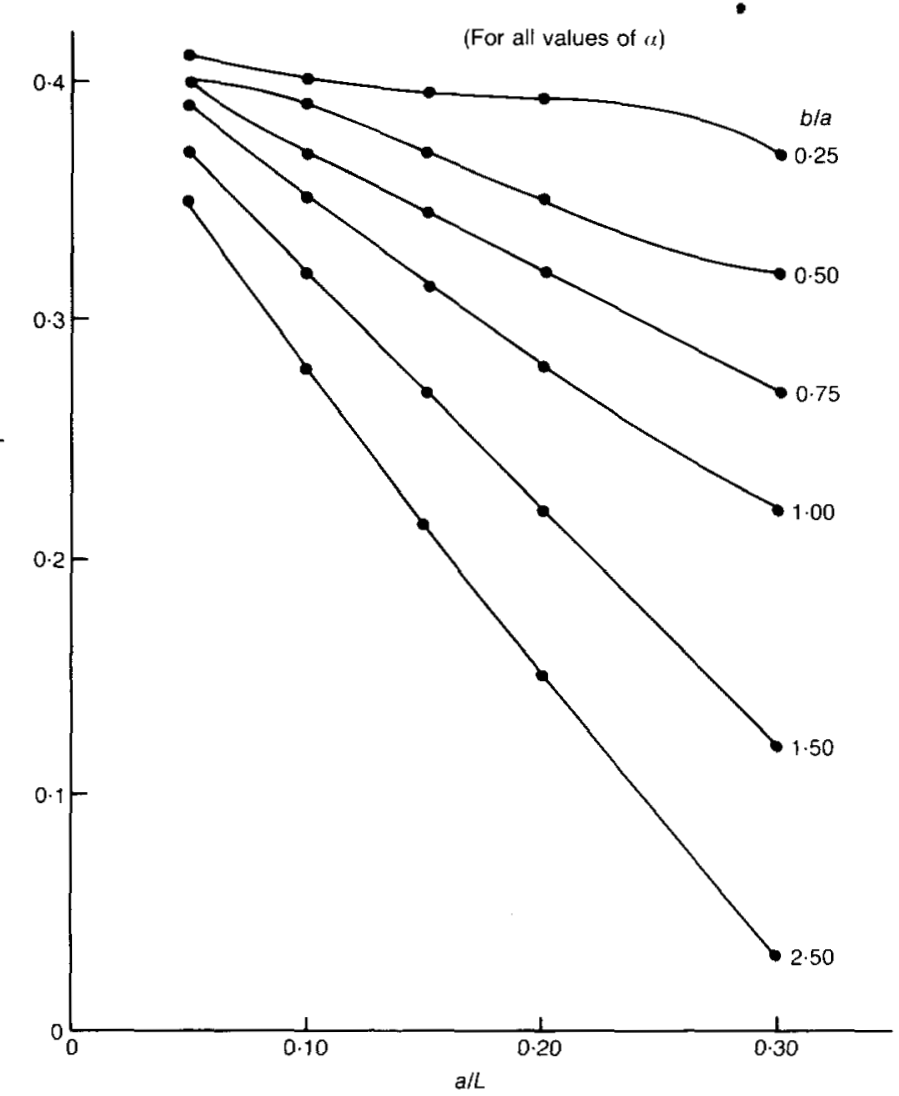

Fig. 10. Variation of $v$ with $a / L$ for flat slabs supported on finite columns 


\section{DISCUSSION}

44. With the moment capacity volume of the strips (Fig. $9(\mathrm{~m})$ ), the moment capacity volume $V_{y 2}$ of the region op' $q^{\prime} \mathrm{r}$ in the $y$ direction is obtained as

$$
\phi_{y 2}=\left[M_{y}^{\prime} L+\bar{M}_{y}^{\prime}(b+v) L\right] a L
$$

The total moment capacity volume of the slab is, therefore, given by

$$
\phi=\mathbb{4}\left(\phi_{x 1}+\phi_{x 2}+\phi_{y 1}+\phi_{y 2}\right)
$$

45. By substituting the expressions for $\phi_{x 1}, \phi_{x 2}$, etc., in equation (50) and simplifying, the following expression for $\phi$ is obtained

$$
\begin{aligned}
\frac{\phi}{4 p L^{4}}= & \frac{(\alpha-a-\beta)^{2}}{2} \alpha+\frac{(1-b-v)^{2}}{2} \alpha \\
& +\alpha(v+b)\left[(1-b-v) v+\frac{v^{2}}{2}+\frac{b(1-b)}{2}\right] \\
& +(a+\beta)\left[(\alpha-\beta-a) \beta+\frac{\beta^{2}}{2}+\frac{(\alpha-a)}{2} \alpha\right]
\end{aligned}
$$

For given values of $a, b$ and $\alpha$, the moment capacity volume $\phi$ is a function of two independent variables $\beta$ and $v$. In order to obtain the minimum moment capacity volume, $\phi$ is minimized with respect to $\beta$ and $v$.

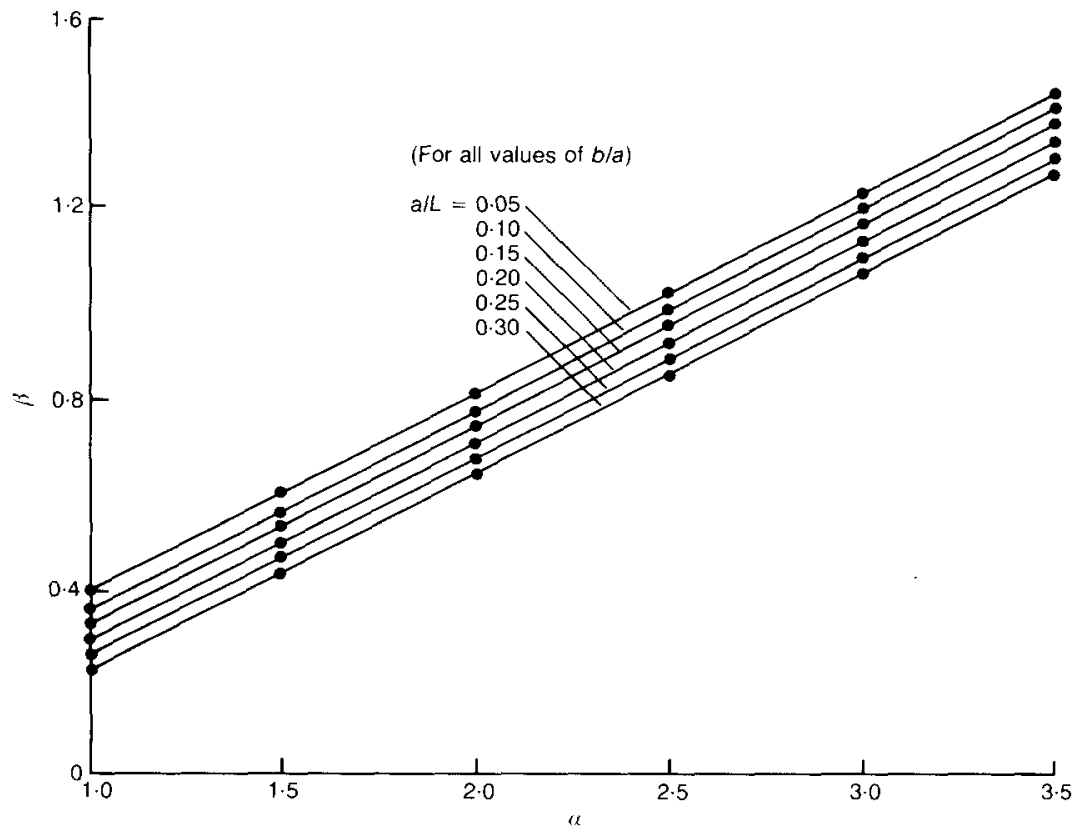

Fig. 11. Variation of parameter $\beta$ with aspect ratio $\alpha$ for flat slabs supported on finite columns 


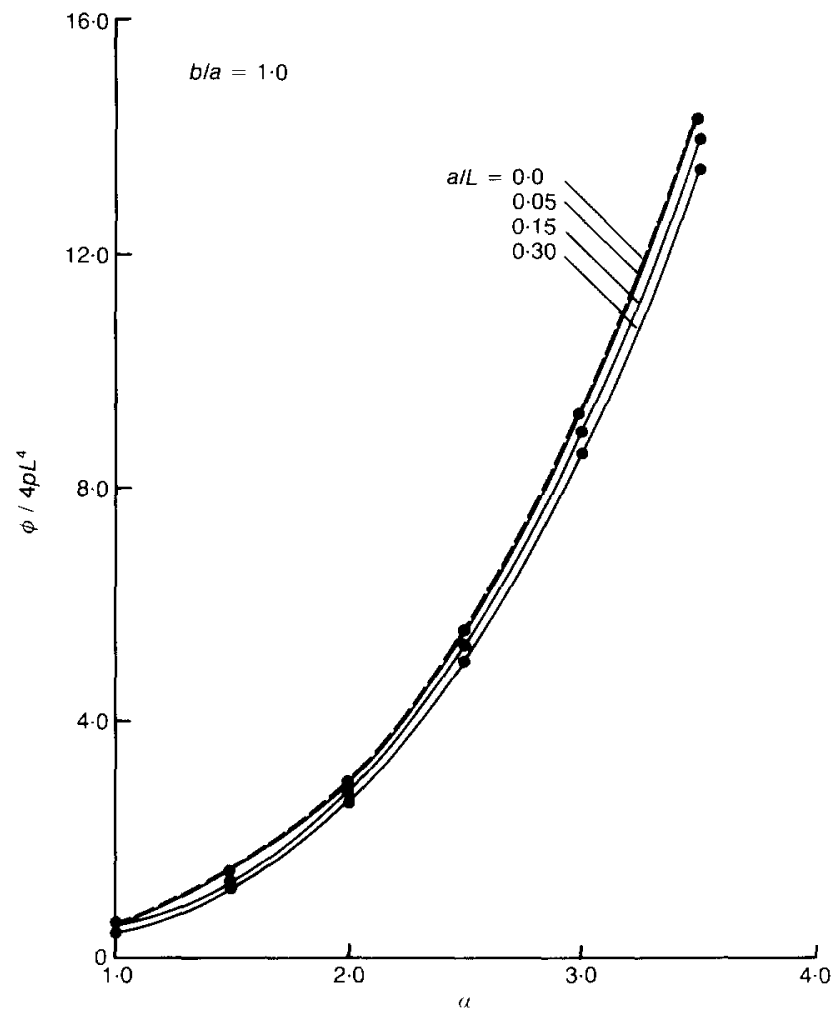

Fig. 12. Variation of minimum moment capacity volume $\left(\phi / 4 p L^{4}\right)$ with aspect ratio $\alpha$ for column supported flat slabs

46. The values of $v$ and $\beta$ for the optimum solution are given in Figs 10 and 11 . Fig. 12 shows the variation of minimum moment capacity volume (non-dimensional) with the aspect ratio for different sizes of finite column supports. It is seen that minimum moment capacity volume does not change significantly with the change in the size of the column. Fig. 12 also shows, by means of a broken line, the variation of the minimum moment capacity volume (exact solution) with the aspect ratio for a point supported slab. The results for the point supported slab are almost the same as those of the finite column supported slab.

\section{References}

16. MelChers R. E. Minimal reinforcement of non-uniform plates. J. Engng Mech. Div. Am. Soc. Civ. Engrs, 1976, 102, No. EM6, Dec., 943-955.

17. Ahmad M. Z. Minimum reinforcement design of r.c. slab. Indian Institute of Technology, New Delhi, 1985, PhD thesis. 